Article

\title{
Tunable Focus Liquid Lens with Radial-Patterned Electrode
}

\section{Miao Xu, Xiahui Wang and Hongwen Ren *}

BK Plus Haptic Polymer Composite Research Team, Department of Polymer Nano Science and Technology, Chonbuk National University, Jeonju, Jeonbuk 561-756, Korea;

E-Mails: xumiao0711@sina.com (M.X.); wangxiahui1986@126.com (X.W.)

* Author to whom correspondence should be addressed; E-Mail: hongwen@jbnu.ac.kr; Tel.: +82-63-270-2354; Fax: +82-63-270-2341.

Academic Editor: Hiroshi Toshiyoshi

Received: 3 July 2015 / Accepted: 11 August 2015 / Published: 17 August 2015

\begin{abstract}
A dielectric liquid lens is prepared based on our previous work. By optimizing the device structure, the liquid lens presents a converging focus with good resolution and changes its focal length over a broad range with a low driving voltage. For a liquid lens with $\sim 2.3 \mathrm{~mm}$ diameter in the relaxed state, it can resolve $\sim 40 \mathrm{lp} / \mathrm{mm}$. The resolution does not degrade during focus change. Its focal length can be varied from $\sim 12$ to $\sim 5 \mathrm{~mm}$ when the applied voltage is changed from 0 to $28 V_{\text {rms }}$. The response time of one cycle is $\sim 2.5 \mathrm{~s}$. Our liquid lens, with a low driving voltage for a large dynamic range, has potential applications in imaging, biometrics, optoelectronic, and lab-on-chip devices.
\end{abstract}

Keywords: adaptive optics; liquid lens; dielectrophoresis; imaging; focal length

\section{Introduction}

Adaptive liquid lenses have potential applications in imaging, optoelectronic, biometrics, and lab-on-chip devices. Various mechanisms were demonstrated [1-17]. In contrast to other liquid lenses, a liquid lens based on the dielectrophoretic effect $[8-10,18]$ is particularly attractive due to the advantages of easy fabrication, compact structure, direct voltage actuation, and low power consumption. Different from an electrowetting lens [1-4], a dielectric liquid lens may not use water as its critical liquid. Therefore, some dielectric liquids with good physical properties can be chosen for improving the lens characteristics without the issues of thermal evaporation and microbubble production. 
Like other liquid lenses, a dielectric liquid lens has its own limitations as well as its strengths. For the previously demonstrated liquid lenses, they mainly face the challenge of a high driving voltage when a broad dynamic focus is changed. For a micro-sized lens, the driving voltage is usually higher than $100 V_{\text {rms }}[9,19]$. To lower the driving voltage, several efforts have been done. One method is to actuate a dielectric liquid lens using a fringing field. Since the fringing field is independent on the cell gap, the liquid lens is scalable. To generate such an electric field, either zone-patterned $[8,10]$ or radial-patterned electrodes [20] can be adopted. In contrast to the former electrode, the latter can provide a continuous field in a radial direction. This field can effectively actuate a liquid lens. However, the lens still needs to be optimized in order to further reduce the driving voltage.

Based on previously-patterned electrodes used for an optical switch [21], here we demonstrated a liquid lens. This patterned electrode can help us improve the performances of our liquid lens. In contrast to a previous liquid lens [20], our liquid lens has the following differences: (1) the width of the electrode strip is unchanged, (2) the gap of adjacent electrode stripes is linearly changed, (3) the number of electrode stripe is largely increased, (4) converging focus rather than diverging focus. Due to the large number of electrode stripes, a low driving voltage can cause a broad dynamic range. The response time is reasonably fast during focus change. Moreover, our lens exhibits the advantages of scalable aperture size, good mechanical stability, and low power consumption.

\section{Device Structure and Mechanism}

When a dielectric liquid droplet is placed in an inhomogeneous electric field, it experiences a dielectric force. The dielectric force can reshape the liquid droplet. To generate an inhomogeneous electric field, an indium-tin-oxide (ITO) electrode is patterned with radial-interdigitated stripes, as depicted in Figure 1a. The ITO stripes connect two annular ITO zones. The two zones have the same center. Half of the stripes connect to the outside zone, while the adjacent stripes connect to the inner zone. The width of each ITO stripe is fixed. Toward the center, the gap of adjacent ITO stripes decreases linearly. The surface of the ITO electrode is covered with a dielectric layer. A sessile liquid droplet is positioned at the center of electrode in the relaxed state. By applying a voltage to the electrode, a fringing field is generated between the adjacent ITO stripes. The fringing field is simply illustrated in Figure $1 b$. Towards the center, the intensity of the electric field increases gradually due to the fan-shaped gap. The electric field is continuous in the radial direction. A dielectric force exerts on the droplet surface is expressed by [22]:

$$
F=-\frac{1}{2} \varepsilon_{0}\left(\varepsilon_{1}-\varepsilon_{2}\right) \nabla E^{2}
$$

where $\varepsilon_{0}, \varepsilon_{1}$, and $\varepsilon_{2}$ represent the permittivity of free space, the dielectric constant of the droplet, and the dielectric constant of the surrounding medium, respectively, and $\nabla E$ denotes the gradient of the electric field. If $\varepsilon_{1}<\varepsilon_{2}$, the force will push the droplet to shift toward the region with the higher electric field. Owing to the circular-symmetrical distribution of the electric field, the dielectric force can contract the liquid droplet at the central area. Since the liquid droplet exhibits a lens character, its focal length can be changed. When the voltage is removed, the dielectric force disappears. The droplet has to recover to its original state due to its interfacial tensions. 


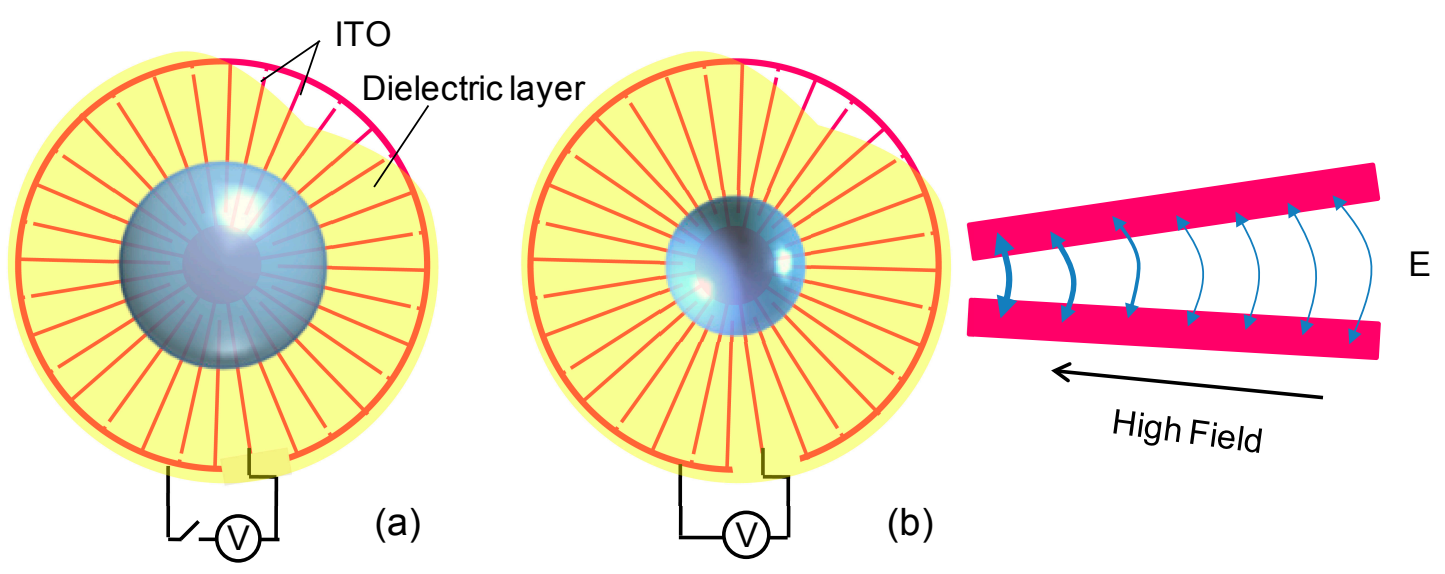

Figure 1. A liquid droplet placed on a radial-patterned electrode in the (a) relaxed state and (b) contracted state. The right chart of (b) shows the fringing field between adjacent ITO stripes.

\section{Experiment}

To get a radial-patterned electrode, an ITO glass substrate is chosen. The ITO is etched with a radial-interdigitated pattern according to the structure of Figure 1a. The size of the electrode substrate is $2 \times 2 \mathrm{~cm}$. Figure 2 a shows partial pattern of the electrode observed using an optical microscope. The slightly red stripes are the remaining ITO electrode. The width of each ITO stripe is $20 \mu \mathrm{m}$, and the gap of adjacent stripes linearly reduces toward the center. The smallest gap is $20 \mu \mathrm{m}$. The diameter of the inner zone is $1 \mathrm{~mm}$. There are 58 stripes connecting to the inner zone and 58 stripes to the outside zone. The substrate surface is coated with a Teflon (400S1-100-1, DuPont, Wilmington, DE, USA) layer. The Teflon layer is used as the dielectric layer which has low surface tension $\left(\gamma_{\mathrm{T}} \sim 19 \mathrm{~N} / \mathrm{m}\right)$ at room temperature [23].

To prepare a liquid lens, a small amount $(\sim 0.57 \mu \mathrm{L})$ of optical oil SL-5267 $\left(\varepsilon_{1} \sim 5, \rho_{\mathrm{g}} \sim 1.26 \mathrm{~g} / \mathrm{cm}^{-3}\right.$, $\left.\gamma_{\mathrm{g}} \sim 50 \mathrm{~N} / \mathrm{m}\right)$ is dripped on the center of the substrate to form a droplet. To balance the gravity effect, glycerol ( $\varepsilon_{2} \sim 47, \rho_{\mathrm{g}} \sim 1.25 \mathrm{~g} / \mathrm{cm}^{-3}, \gamma_{\mathrm{g}} \sim 63 \mathrm{~N} / \mathrm{m}$ ) with high purity $(>99.5 \%)$ is chosen as the liquid to fill the surroundings of the droplet. The two liquids are covered with a top glass substrate to form a cell, as depicted in Figure 2 b. The cell gap controlled by glass stripes is thick enough so that the dome of the droplet could not touch the top substrate. The periphery of the cell is tightly sealed with glue. In the relaxed state, the diameter of the droplet on the bottom glass substrate is $\sim 2.3 \mathrm{~mm}$.
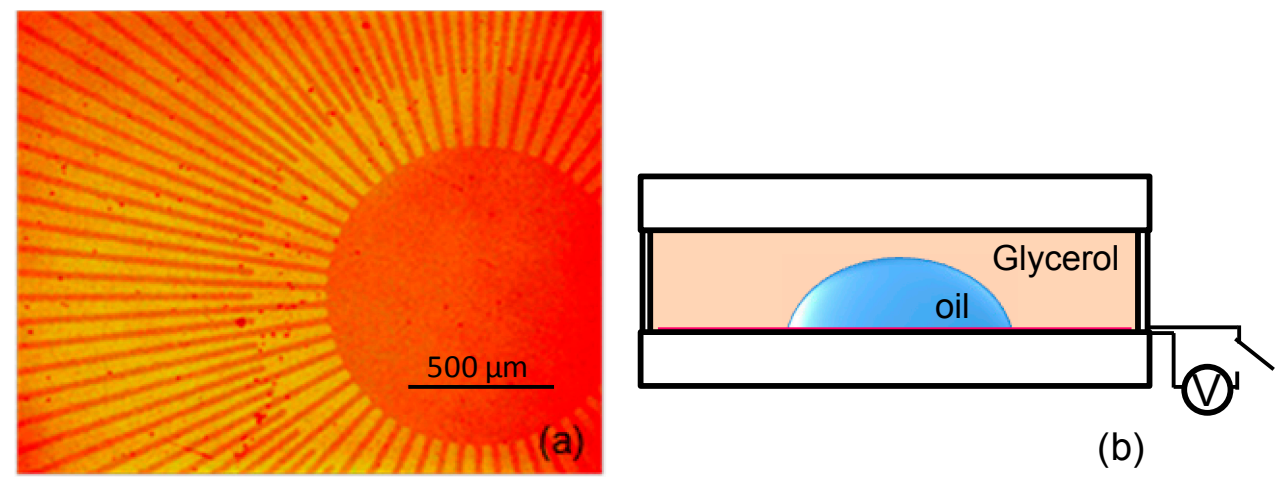

(b)

Figure 2. (a) Image of partial ITO pattern and (b) side-view structure of the lens cell. 


\section{Results and Discussion}

The refractive index of the oil droplet $\left(n_{1} \sim 1.67\right)$ is larger than that of the surrounding glycerol $\left(n_{2} \sim 1.47\right)$, so the liquid lens is a converging lens. To characterize the performance of the lens, we observe the image of an object through the lens when it is actuated by a voltage. A Doraemon toy is chosen as the object. The object is placed under the lens cell at $\sim 13 \mathrm{~cm}$. A digital camera is placed above the lens cell to record the image. In the relaxed state $(V=0)$, a clear image is observed in white light, as shown in Figure 3a. The image is inverted in contrast to the position of the object. To actuate the liquid lens, a voltage $(500 \mathrm{~Hz})$ is applied to the electrode. When the voltage exceeds $V=18 V_{\mathrm{rms}}$, the image becomes slightly blurry, as shown in Figure $3 \mathrm{~b}$. This voltage is the threshold voltage of the liquid lens. The blurry image implies that the focal length of the liquid lens is changed. The focal length of the liquid lens is expressed by:

$$
f=\frac{r}{n_{1}-n_{2}}
$$

where $r$ is the radius of the droplet curvature. According to Figure $1 b$, the radius $r$ of the droplet curvature is decreased when the droplet is actuated. As a result, its focal length $f$ is reduced.
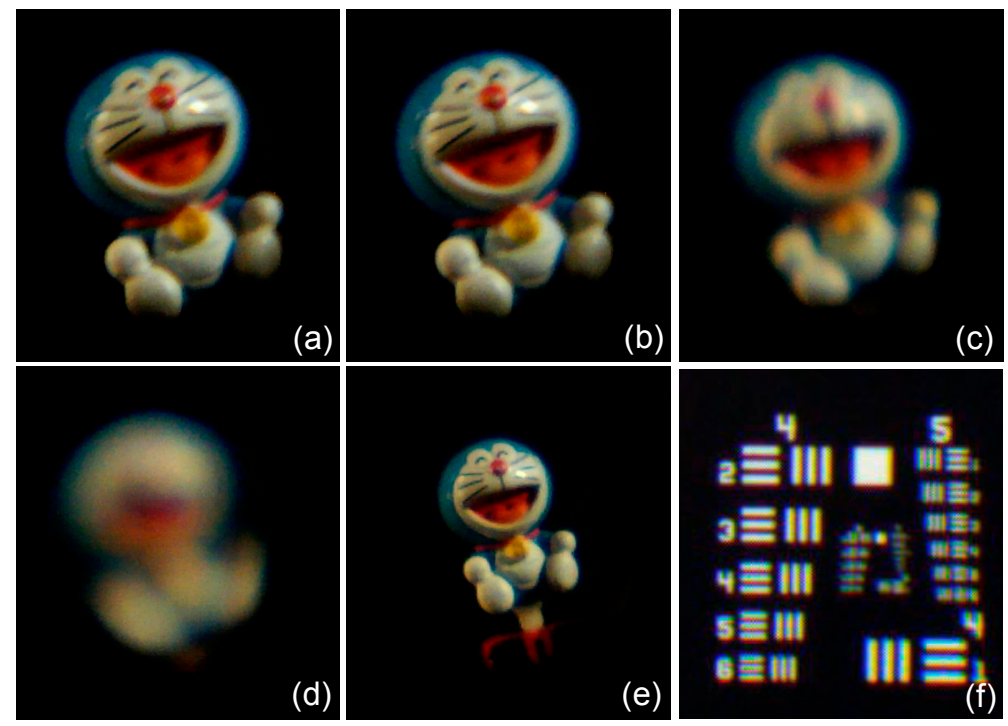

Figure 3. Image of a toy observed through the liquid lens at various voltages. (a) $V=0$, (b) $V=18 V_{\text {rms }}$, (c) $V=30 V_{\text {rms }}$, (d) $V=45 V_{\text {rms }}$, and (e) refocus at $V=45 V_{\text {rms }}$, (f) image of resolution target at $V=0$.

When the voltage is increased gradually, the image becomes much more blurry. Figure $3 \mathrm{c}, \mathrm{d}$ show the images when $V=30 V_{\text {rms }}$ and $45 V_{\text {rms }}$, respectively. When $V=45 V_{\text {rms }}$, the image is highly blurry and could not be recognized completely due to the large defocus. To get a clear image at $V=45 V_{\text {rms }}$, the lens cell is shifted toward the camera. A refocused image with reduced size is observed, as shown in Figure 3e. To estimate the resolution of the lens, a resolution target is used to replace the toy. In the relaxed state, our eye can distinguish group 5 and element 3 of the target. The corresponding resolution of the lens is $\sim 40 \mathrm{lp} / \mathrm{mm}$. To record the image with a CCD, a glass lens is used in order to sufficiently magnify the image. The image of the resolution target is shown in Figure $3 \mathrm{f}$. It could resolve group 5 
and element 2, and the corresponding resolution is $\sim 36 \mathrm{lp} / \mathrm{mm}$. Due to the aberration of the added glass lens, the observed resolution degrades slightly.

Suppose the lens has no aberration, so the resolution of the lens is limited by diffraction. The limiting resolution can be expressed by [24,25]:

$$
\mathrm{R}_{(\mathrm{c})} \approx \frac{d}{S \lambda}
$$

where $d$ is the diameter of the lens aperture, $s$ is the distance of the object, and $\lambda$ is the wavelength of light. For $s=13 \mathrm{~cm}, \lambda=0.5 \mu \mathrm{m}$, and the diameter of the lens aperture $d \sim 2.3 \mathrm{~mm}$, the diffraction-limited resolution is calculated to be $\sim 44 \mathrm{lp} / \mathrm{mm}$. As a comparison, the observed resolution $(\sim 40 \mathrm{lp} / \mathrm{mm})$ by an eye is lower than that of the diffraction-limited resolution due to the lens aberration. Since this resolution is close to the diffraction-limited resolution, the diffraction limit plays the main role rather than the spherical aberration. When the diameter of the lens $d$ is decreased, the shape of the droplet becomes contracted. To get a clear image, the object distance $s$ should be decreased. As a result, the observed resolution is insensitive to the shape change of the liquid lens.

Due to the low surface tension, the droplet has a contact angle on the Teflon surface. The contact angle $\theta$ is defined geometrically as the angle formed by the droplet at the three-phase boundary where the droplet, the glycerol, and the Teflon layer intersect. When the droplet is reshaped by a voltage, its contact angle is changed. To measure the voltage-dependent contact angle, one simple method is to monitor the shape of liquid droplet. The digital camera is used to record the side-view surface profile of the droplet. The voltage-dependent shape change of the droplet is shown in Figure 4. In relaxed state, the radius of the droplet curvature is the largest. When a voltage applied to the electrode exceeds $18 V_{\text {rms }}$, the shape of the droplet begins to change. As the voltage increases gradually, the droplet bulges more and more. When the voltage is higher than $45 V_{\text {rms }}$, the shape of the droplet does not change obviously. The liquid lens driven with a relatively low voltage is mainly due to two factors: (1) the gap of adjacent stripes is linearly decreased and (2) the voltage is increased. The two factors can largely increase the fringing field or the generated dielectric force, causing the liquid droplet to be deformed effectively. To visually observe the shape change of the droplet, a gated square-wave of $45 V_{\text {rms }}$ pulse is applied to the electrode. A dynamic video (Media 1) is recorded as given in Figure 4. The shape change of the droplet is relatively fast.

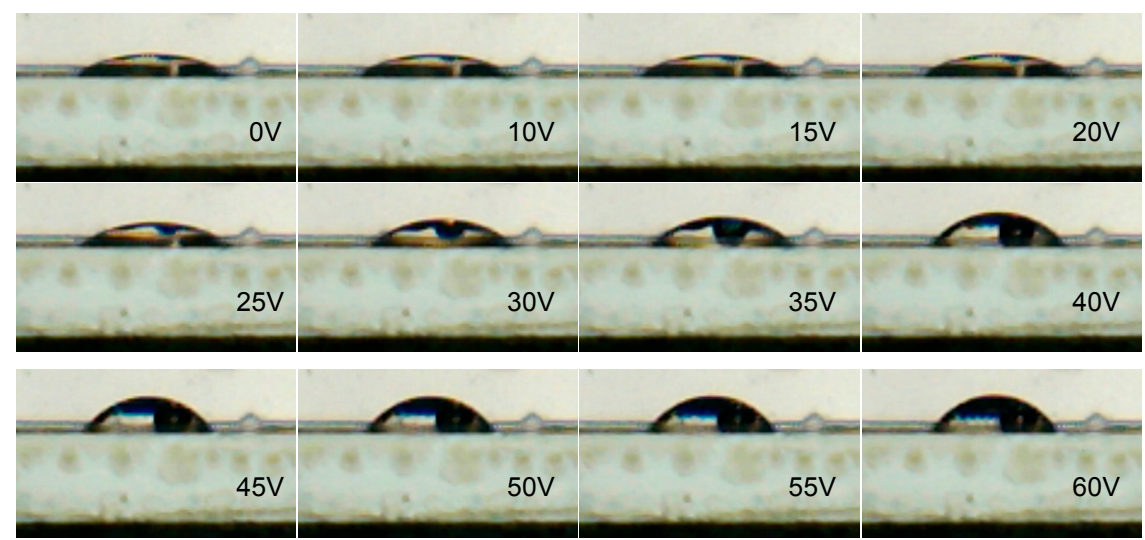

Figure 4. Voltage-dependent shape change of the liquid droplet. A dynamic video showing the shape change of the droplet is given at $V=45 V_{\text {rms }}$. 
According to the shape change of the droplet shown in Figure 4, the contact angle $\theta$ of the droplet and the diameter of the droplet aperture at various voltages are measured. The results are given in Figure 5. When $V=0, \theta$ is measured to be $\sim 27.8^{\circ}$. The contact angle varies from $27.8^{\circ}$ to $55.8^{\circ}$ when the voltage

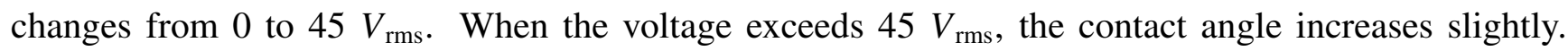
When contact angle increases, the diameter of the droplet aperture decreases due to the fixed volume. The diameter varies from $\sim 2.3$ to $\sim 1.7 \mathrm{~mm}$ when the voltage changes from 0 to $50 V_{\text {rms. }}$.

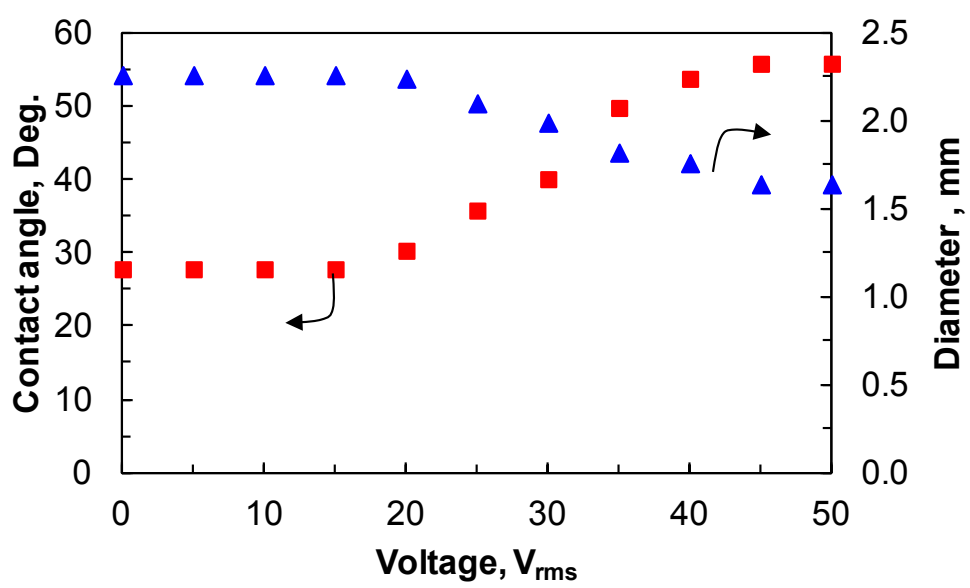

Figure 5. The contact angle and the diameter of the droplet aperture changed with voltages.

To measure the focal length of the liquid lens, a collimated He-Ne laser beam $(\lambda \sim 633 \mathrm{~nm})$ is used to illuminate the liquid lens. The beam passing through the lens is focused at a spot. The distance from the focal spot to the lens is the focal length. Figure 6 shows the focal length changed with different voltages (triangles). Due to the thin waist of the laser beam, it is difficult to precisely locate the focal point. As a result, error bars are given.

Using the measured contact angle (Figure 4), it is convenient to theoretically calculate the focal length of the liquid lens as well. The relationship of the contact angle and the focal length of the lens can be expressed by [3]:

$$
f=\frac{3 V}{\pi(1-\cos \theta)\left(2-\cos ^{2} \theta-\cos \theta\right)\left(n_{1}-n_{2}\right)^{3}}
$$

where $V$ is the volume of the droplet. From Equation (4), changing the contact angle $\theta$ will vary the focal length $f$ of the liquid lens or vice versa.

The calculated focal length is also given in Figure 6 (dots). When the voltage changes from 0 to $50 V_{\text {rms }}$, the focal length varies from $\sim 12$ to $\sim 5 \mathrm{~mm}$. The calculated focal length and the measured focal length overlap well in the range of error bars.

For dynamic actuation, the response time of the liquid lens is measured as well. To measure the response time, a He-Ne laser beam is used to illuminate the liquid lens. A photodiode detector is placed behind the lens cell to receive the laser beam. In the relaxed state, part of the beam is received by the detector when it is placed in a defocusing position. A digital oscilloscope is connected to the detector. The beam intensity change with time is analyzed by the oscilloscope. When a voltage is applied to the lens cell, the beam is focused at the position of the detector, and more light is received by the detector. Figure 7 shows the time dependent light intensity when a gated square-wave of $28 V_{\text {rms }}$ pulse is applied 
to the cell. The duration time is $5 \mathrm{~s}$. It takes $\sim 1 \mathrm{~s}$ for the droplet to reshape to a stable state and $\sim 1.5 \mathrm{~s}$ for the reshaped droplet to recover to its original state. The driving cycle with four periods shows that the liquid lens has good mechanical stability. For one driving cycle, the power consumption of the lens is about hundred micro-watts.

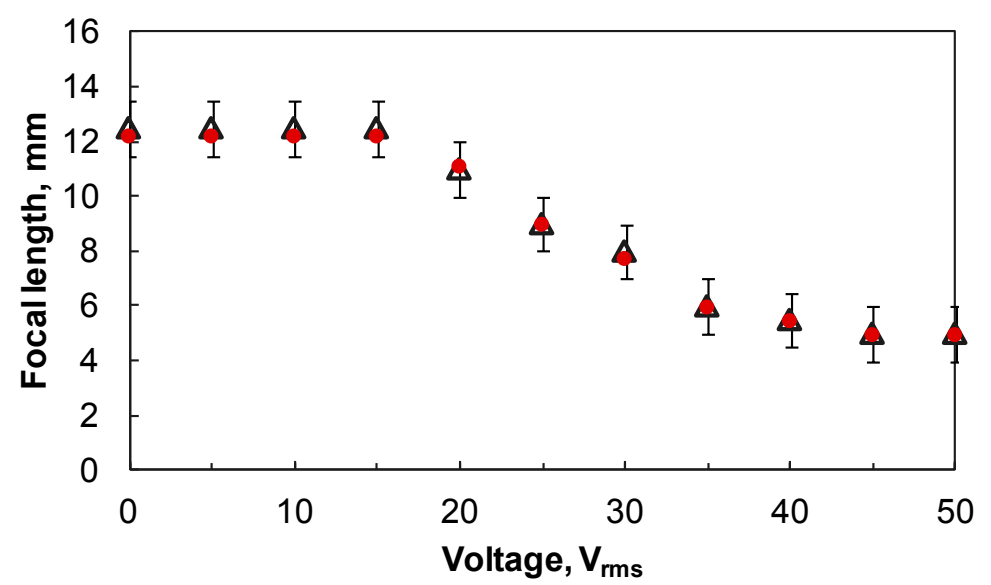

Figure 6. The measured focal length (triangles) and the calculated focal length (dots) of the liquid lens changed with voltages.

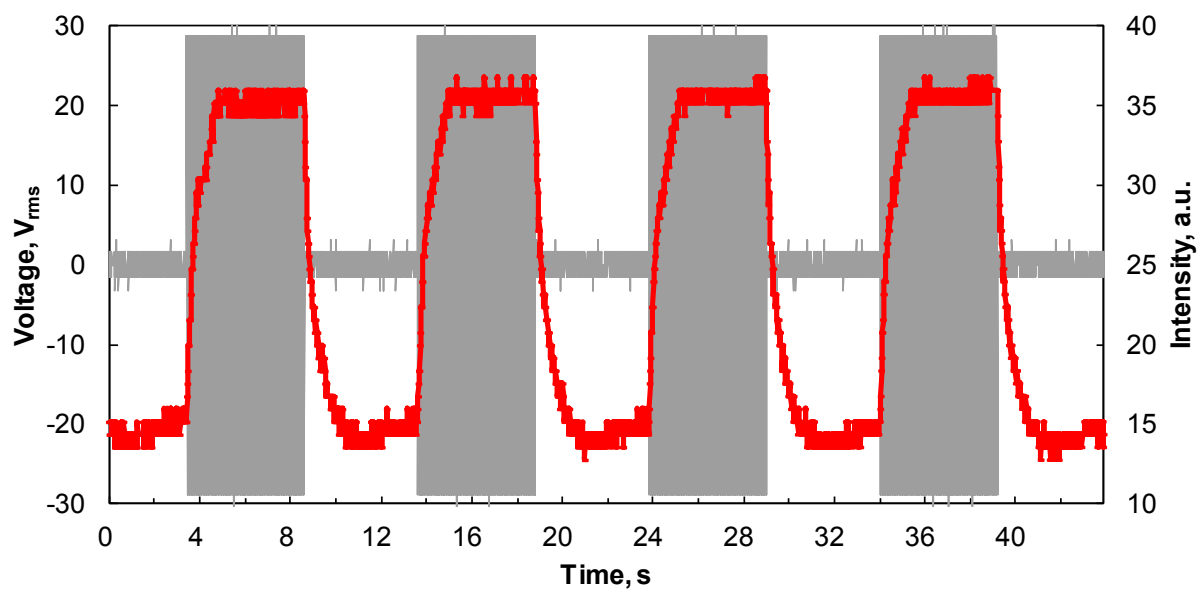

Figure 7. Dynamic response of the liquid lens impacted by voltage pulses.

Like previous liquid lenses, the size of the liquid droplet is insensitive to the driving voltage because of the fringing field actuation on one side of the substrate. Therefore, the size of the liquid lens is scalable. The driving voltage can be further reduced by increasing the number of ITO stripes. The densities of the two liquids match well, so the gravity effect is negligible when the lens cell is placed in a vertical direction. The liquid lens is also mechanically stable with no shocking or vibrating effects. The droplet can be fixed on the Teflon surface due to the adhesive force. Since the radial-interdigitated electrode provides a circularly-symmetrical electric field, the droplet can be uniformly reshaped without distortion. Due to this reason, our lens can present high resolution $(\sim 40 \mathrm{lp} / \mathrm{mm})$ during the focus change. Owing to the radial-interdigitated electrode, the generated dielectric force can effectively reshape the liquid droplet. This is the reason why our liquid lens needs a relatively low driving voltage $\left(<45 V_{\text {rms }}\right)$ for a large dynamic range. The response time is reasonably fast when a limited focus change is required. 


\section{Conclusions}

A dielectric liquid lens is fabricated using a newly designed electrode. Different from previous approaches, the radial-interdigitated ITO stripes can provide a continuous fringing field in the radial direction. The electric field can effectively deform the shape of the liquid droplet with a relatively low voltage. Using 58/58 ITO stripes in the radial-interdigitated pattern, the focal length of $2.3 \mathrm{~mm}$ diameter liquid lens can be tuned from $\sim 12$ to $\sim 5 \mathrm{~mm}$ when the voltage is changed from 0 to $45 V_{\text {rms. The }}$ resolution of the lens can resolve up to $\sim 40 \mathrm{lp} / \mathrm{mm}$ in its relaxed state. The resolution will not degrade during dynamic actuation. The response time is about $\sim 2.5 \mathrm{~s}$ in one driving cycle. Like the previous liquid lenses, the size of our liquid lens is scalable. The lens also has good mechanical stability because the densities of the two liquids match well. Owing to these advantages, our liquid lens has potential applications in biometrics, optical communication, machine vision, and cell phones.

\section{Supplementary Materials}

Supplementary materials can be accessed at: http://www.mdpi.com/2072-666X/6/8/1157/s1.

\section{Acknowledgments}

This work is financially supported by the National Research Foundation of Korea under Grant 2014064156.

\section{Author Contributions}

Miao $\mathrm{Xu}$ conceived and designed the experiments; Miao $\mathrm{Xu}$ and Xiahui Wang performed the experiments; Hongwen Ren analyzed the data; Miao $\mathrm{Xu}$ and Xiahui Wang contributed reagents/materials/analysis tools; Hongwen Ren and Miao Xu wrote the paper together.

\section{Conflicts of Interest}

The authors declare no conflict of interest.

\section{References}

1. Berge, B.; Peseux, J. Variable focal lens controlled by an external voltage: An application of electrowetting. Eur. Phys. J. E 2000, 3, 159-163. [CrossRef]

2. Krupenkin, T.; Yang, S.; Mach, P. Tunable liquid microlens. Appl. Phys. Lett. 2003, 82, 316-318.

3. Kuiper, S.; Hendriks, B.H.W. Variable-focus liquid lens for miniature cameras. Appl. Phys. Lett. 2004, 85, 1128-1130. [CrossRef]

4. Grilli, S.; Miccio, L.; Vespini, V.; Finizio, A.; de Nicola, S.; Ferraro, P. Liquid micro-lens array activated by selective electrowetting on lithium niobate substrates. Opt. Express. 2008, 16, 8084-8093. [CrossRef] [PubMed]

5. Chronis, N.; Liu, G.L.; Jeong, K.H.; Lee, L.P. Tunable liquid-filled microlens array integrated with microfluidic network. Opt. Express. 2003, 11, 2370-2378. [CrossRef] [PubMed] 
6. Wang, L.; Oku, H.; Ishikawa, M. Variable-focus lens with $30 \mathrm{~mm}$ optical aperture based on liquidmembrane-liquid structure. Appl. Phys. Lett. 2013, 102, 131111. [CrossRef]

7. Dong, L.; Agarwal, A.K.; Beebe, D.J.; Jiang, H. Adaptive liquid microlenses activated by stimuli-responsive hydrogels. Nature 2006, 442, 551-554. [CrossRef] [PubMed]

8. Cheng, C.-C.; Yeh, J.A. Dielectrically actuated liquid lens. Opt. Express. 2007, 15, 7140-7145.

9. Ren, H.; Xianyu, H.; Xu, S.; Wu, S.T. Adaptive dielectric liquid lens. Opt. Express. 2008, 16, 14954-14960. [CrossRef] [PubMed]

10. Yang, C.C.; Tsai, C.-W.G.; Yeh, J.A. Dynamic behavior of liquid microlenses actuated using dielectric force. J. Microelectromech. Syst. 2011, 20, 1143-1149. [CrossRef]

11. Koyama, D.; Isago, R.; Nakamura, K. Compact, high-speed variable-focus liquid lens using acoustic radiation force. Opt. Express. 2010, 18, 25158-25169. [CrossRef] [PubMed]

12. Cheng, H.C.; Xu, S.; Liu, Y.; Levi, S.; Wu, S.T. Adaptive mechanical-wetting lens actuated by ferrofluids. Opt. Commun. 2011, 284, 2118-2121. [CrossRef]

13. Carpi, F.; Frediani, G.; Turco, S.; Rossi, D.R. Bioinspired tunable lens with muscle-like electroactive elastomers. Adv. Funct. Mater. 2011, 21, 4152-4158. [CrossRef]

14. Shian, S.; Diebold, R.M.; Clarke, D.R. Tunable lenses using transparent dielectric elastomer actuators. Opt. Express. 2013, 21, 8669-8676. [CrossRef] [PubMed]

15. Wei, K.; Domicone, N.W.; Zhao, Y. Electroactive liquid lens driven by an annular membrane. Opt. Lett. 2014, 39, 1318-1321. [CrossRef] [PubMed]

16. Maffli, L.; Rosset, S.; Ghilardi, M.; Carpi, F.; Shea, H. Ultrafast all-polymer electrically tunable silicone lenses. Adv. Funct. Mater. 2015, 25, 1656-1665. [CrossRef]

17. Malouin, B.A., Jr.; Vogel, M.J.; Olles, J.D.; Cheng, L.; Hirsa, A.H. Electromagnetic liquid pistons for capillarity-based pumping. Lab Chip 2011, 11, 393-397. [CrossRef] [PubMed]

18. Lu, Y.S.; Tu, H.; Xu, Y.; Jiang, H. Tunable dielectric liquid lens on flexible substrate. Appl. Phys. Lett. 2013, 103, 261113. [CrossRef] [PubMed]

19. Hung, K.Y.; Fan, C.C.; Tseng, F.G.; Chen, Y.K. Design and fabrication of a copolymer aspheric bi-convex lens utilizing thermal energy and electrostatic force in a dynamic fluidic. Opt. Express. 2010, 18, 6014-6023. [CrossRef] [PubMed]

20. Xu, M.; Xu, D.; Ren, H.; Yoo, I.S.; Wang, Q.H. An adaptive liquid lens with radial interdigitated electrode. J. Opt. 2014, 16, 105601. [CrossRef]

21. Xu, M.; Ren, H.; Lin, Y.H. Electrically actuated liquid iris. Opt. Lett. 2015, 40, 831-834.

22. Penfield, P.; Haus, H.A. Electrodynamics of Moving Media; MIT press: London, UK, 1967.

23. Kim, B.H.; Lee, D.H.; Kim, J.Y.; Shin, D.O.; Jeong, H.Y.; Hong, S.; Yun, J.M.; Koo, C.M.; Lee, H.; Kim, S.O. Mussel-inspired block copolymer lithography for low surface energy materials of Teflon, grapheme, and gold. Adv. Mater. 2011, 23, 5618-5622. [CrossRef] [PubMed]

24. Ren, L.; Lee, R.H.; Park, H.R.; Ren, H.; Nah, C.; Yoo, I.S. A liquid lens driven by bubble actuator. J. Microelectromech. Syst. 2013, 22, 1222-1228. [CrossRef]

25. Smith, W.J. Modern Optical Engineering, 2rd ed.; McGraw-Hill: New York, NY, USA, 2000.

(C) 2015 by the authors; licensee MDPI, Basel, Switzerland. This article is an open access article distributed under the terms and conditions of the Creative Commons Attribution license (http://creativecommons.org/licenses/by/4.0/). 